\title{
The impacts of maternal iron deficiency and being overweight during pregnancy on neurodevelopment of the offspring
}

\author{
Staffan K. Berglund ${ }^{1,2}$, Francisco J. Torres-Espínola ${ }^{1}$, Luz García-Valdés ${ }^{1}$, M $^{a}$ Teresa Segura $^{1}$, \\ Cristina Martínez-Zaldívar ${ }^{1}$, Carmen Padilla ${ }^{3}$, Ricardo Rueda ${ }^{4}$, Miguel Pérez García ${ }^{5}$, Harry J. McArdle ${ }^{6}$ \\ and Cristina Campoy ${ }^{1,7,8 *}$ \\ ${ }^{1}$ EURISTIKOS Excellence Centre for Paediatric Research, University of Granada, 18016 Granada, Spain \\ ${ }^{2}$ Department of Clinical Sciences, Pediatrics, Umeå University, 90185 Umeå, Sweden \\ ${ }^{3}$ Clinical Service of Obstetrics and Gynaecology, Clinical University Hospital San Cecilio, 18012 Granada, Spain \\ ${ }^{4}$ Abbott Laboratories, RED Department, 18004 Granada, Spain \\ ${ }^{5}$ Department of Clinical Neuropsychology, Mind, Brain and Behavior Research Centre, University of Granada, 18016 Granada, Spain \\ ${ }^{6}$ The Rowett Institute of Nutrition and Health, University of Aberdeen, Aberdeen AB21 9SB, UK \\ ${ }^{7}$ Department of Paediatrics, University of Granada, 18004 Granada, Spain \\ ${ }^{8}$ CIBER of Epidemiology and Public Health (CIBERESP), 18004 Granada, Spain
}

(Submitted 29 September 2016 - Final revision received 6 August 2017 - Accepted 15 August 2017)

\begin{abstract}
Both maternal Fe deficiency (ID) and being overweight or obese (Ow/Ob, BMI $\geq 25 \mathrm{~kg} / \mathrm{m}^{2}$ ) may negatively affect offspring brain development. However, the two risk factors correlate and their independent effects on infant neurodevelopment are unclear. PREOBE is a prospective observational study that included 331 pregnant Spanish women, of whom 166 had pre-gestational Ow/Ob. Fe status was analysed at 34 weeks and at delivery, and babies were assessed using Bayley III scales of neurodevelopment at 18 months. In confounder-adjusted analyses, maternal ID at 34 weeks was associated with lower composite motor scores at 18 months (mean 113.3 (SD 9.9) $v .117 \cdot 1$ (SD 9.2), $P=0.039)$. Further, the offspring of mothers with ID at delivery had lower cognitive scores (114.0 (sD 9.7) $v$. 121.5 (SD 10.9), $P=0 \cdot 039)$ and lower receptive, expressive and composite (99.5 (sD 8.6) v. 107.6 (sD 8.3), $P=0.004$ ) language scores. The negative associations between maternal ID at delivery and Bayley scores remained even when adjusting for maternal Ow/Ob and gestational diabetes. Similarly, maternal Ow/Ob correlated with lower gross motor scores in the offspring (12.3 (SD 2.0) $v .13 \cdot 0$ (SD 2.1), $P=0 \cdot 037$ ), a correlation that remained when adjusting for maternal ID. In conclusion, maternal ID and pre-gestational Ow/Ob are both negatively associated with Bayley scores at 18 months, but independently and on different subscales. These results should be taken into account when considering Fe supplementation for pregnant women.
\end{abstract}

Key words: Iron: Overweight: Obesity: Infant neurodevelopment: Bayley test

The importance of the nutritional environment on brain development during fetal life and, particularly, during the third trimester has earned increasing interest ${ }^{(1-4)}$. Several essential nutrients are now considered critical for both short- and longterm neurodevelopment of the unborn child. One such nutrient is $\mathrm{Fe}$. $\mathrm{Fe}$ is essential for synaptogenesis, myelination and nerve-cell metabolism, and Fe deficiency (ID) during fetal or in early postnatal life is an established risk factor for poor neurodevelopment ${ }^{(1,5-7)}$. Several groups that are at a risk for being $\mathrm{Fe}$ deficient, such as low-birth weight babies, babies born to diabetic mothers or children above 6 months of age with low $\mathrm{Fe}$ intake, have been studied in relation to their neurodevelopment, with negative associations found ${ }^{(8-11)}$. Another possible risk group of prenatal or neonatal ID are the offspring of mothers with poor Fe status ${ }^{(12,13)}$. Even though the placenta has an effective regulatory ability for Fe transfer, maternal ID has been associated with lower Fe stores in the fetus ${ }^{(12-17)}$. However, few studies have explored the direct correlation between maternal ID and infant neurodevelopment, and the overall risk of adverse neurodevelopment following maternal ID has been considered low. Therefore, at least in developed countries, general Fe supplementation is currently not recommended ${ }^{(18,19)}$.

Other fetal risk factors also associated with impaired neurodevelopment include maternal metabolic pathologies

Abbreviations: GD, gestational diabetes; ID, Fe deficiency; IDA, Fe deficiency anaemia; Ow/Ob, overweight or obese.

* Corresponding author: Professor C. Campoy, email ccampoy@ugr.es 
such as being overweight (Ow), obesity (Ob) and gestational diabetes (GD). Offspring of mothers with these conditions show poorer long-term development of memory, motor development and overall cognition ${ }^{(20-23)}$. However, the mechanisms are unclear and causality is not yet determined; thus, confounding effects may possibly explain the associations. Interestingly, obesity is also known as a risk factor for ID, most likely caused by an increased inflammatory status and, consequently, by an up-regulation of the Fe-regulatory protein hepcidin ${ }^{(24-27)}$. This increased risk for ID in obese mothers may be a contributing confounder to the associations observed between maternal metabolic pathologies and neurodevelopment. It has recently been suggested that babies born to obese mothers may have reduced Fe stores, further supporting a link between the two observed groups of risk factors ${ }^{(28)}$.

The PREOBE study is a prospective observational study with the general objective of studying the perinatal and postnatal influence of maternal $\mathrm{Ow}, \mathrm{Ob}$ and GD on growth and neurodevelopment in the offspring, compared with healthy normalweight controls ${ }^{(29)}$. In previous analyses from this cohort, we confirmed that obese women have an increased risk for ID ${ }^{(29)}$. Further, we have observed differences in the neurodevelopmental profile of the offspring of obese mothers ${ }^{(30)}$

The objectives of the current secondary analyses were to explore whether maternal ID and $\mathrm{Ow} / \mathrm{Ob}$ are independently associated with neurodevelopment at 18 months of age in the offspring from the PREOBE cohort.

\section{Methods}

\section{Study design and participants}

The methodology of the present study has been published in detail elsewhere ${ }^{(29)}$. In brief, we included 331 women, pregnant between 2008 and 2012, through a collaboration with the tertiary 'San Cecilio' University Clinical Hospital and the MotherInfant University Hospital in Granada (Spain). The inclusion criteria were singleton pregnancies, maternal age between 18 and 45 years, no simultaneous participation in any other research study, no kind of drug treatment, no diagnosed diseases other than obesity, Ow or GD (e.g. pre-GD or hypertension) and no unusual or vegan diets. Exclusion criteria used in the current analyses were preterm birth $(<37$ weeks of gestation) and congenital disorders in the offspring. As described elsewhere, we actively recruited women with different pre-gestational BMI including those of normal weight $\left(18.5 \mathrm{~kg} / \mathrm{m}^{2} \leq \mathrm{BMI}<25 \mathrm{~kg} / \mathrm{m}^{2}\right)$, those who were Ow $\left(25 \mathrm{~kg} / \mathrm{m}^{2} \leq \mathrm{BMI}<30 \mathrm{~kg} / \mathrm{m}^{2}\right)$ and those with obesity (BMI $\geq 30 \mathrm{~kg} / \mathrm{m}^{2}$ ). Further, we actively included mothers from all three BMI categories who were diagnosed with GD. The majority of mothers ( $n$ 269) were approached and included at a gestational age of 12-20 weeks, during a screening visit at the obstetric clinic. However, to increase the number of included mothers diagnosed with GD, an additional recruitment of mothers ( $n$ 62) was performed during a similar visit at a gestational age of $24-34$ weeks ${ }^{(29)}$. The overall cohort included 166 women with pre-gestational Ow/Ob. A total of forty-six women had or were diagnosed with GD at 34 weeks of gestation. The remaining 165 cases had normal pre-gestational BMI. Of these, thirty-three had GD (Fig. 1).

The clinical practice for pregnant women in Spain follows recommendations from the Spanish society for gynaecology and obstetrics (www.sego.es). According to these recommendations, mothers diagnosed as being $\mathrm{Ow} / \mathrm{Ob}$ are given weight-reducing nutritional advice. Further, mothers diagnosed with GD are routinely referred to an endocrinologist and a nutritionist, based on which they are given nutritional and lifestyle recommendations and, in some cases, insulin treatment to optimise glucose control ${ }^{(31)}$. Mothers included in the present study continued their ongoing regular check-ups and possible treatments at the obstetric and endocrinology clinic, in parallel with the data collection for the present study.

This study was conducted according to the guidelines laid down in the Declaration of Helsinki and all procedures were approved by the Bioethical Committees for Clinical Research of the Clinical University Hospitals of Granada. Ethical approval was also obtained from the Research Bioethical Committee of the University of Granada. Written informed consent was obtained from all participants at study entry. The project was registered at www.ClinicalTrial.gov no.: NCT01634464.

\section{Maternal and perinatal data collection}

The details of the protocol were described previously ${ }^{(29)}$. During the screening visit at a gestational age of 12-20 weeks (or 24-34 weeks for the sixty-two mothers recruited in late pregnancy), and after signing the informed consent form, the mothers' height and pre-gestational weight were measured. A questionnaire about the sociodemographic background (education, age, number of previous children, etc.) and ongoing Fe supplementation (present or not) was administered. The pre-gestational weight was self-reported, whereas the height was measured and the information was used to calculate the pre-gestational BMI. Thereafter, the mothers were invited for a visit at 24 and 34 weeks of gestation and were also assessed, whenever possible, at delivery. The data collection from the visit at 24 weeks is not included in the present analyses. The visit at 34 weeks of gestation and the assessment at delivery included anthropometric measurements and blood collection by venous puncture. Cord blood from the newborn infant was collected and analysed in all cases when it was available. Perinatal data were registered including mode of delivery, Apgar score, gestational age at birth, infant anthropometrics and any admission to the neonatal ward. Routine maternal checkups in regular medical care - for example, $\mathrm{Hb}$ tests - were performed at the obstetric clinic in parallel with the present study and not monitored in detail.

At 3 months, the infant feeding mode was categorised by a paediatrician into exclusive breast-feeding, exclusive formula feeding or mixed feeding when breast milk and formula were combined.

Maternal and cord blood were analysed at the University Hospital laboratory for $\mathrm{Hb}$ and mean cell volume (MCV) using flow cytometry (ADVIA 2120; Siemens HealthCare); ferritin and transferrin were analysed using an automatic analyser (Elecsys 2010 with modular analytics E170; Roche); and Fe was analysed 
using enzyme-colorimetric automated methods for clinical chemistry (Modular Analytics EVO; Roche). Transferrin saturation (TS) was calculated using the formula: TS $(\%)=($ ferritin $(\mu \mathrm{g} / \mathrm{ml}) \times 100) /($ transferrin $(\mathrm{mg} / \mathrm{dl}) \times 1 \cdot 24)$. Maternal Fe-deficient erythropoiesis (ID) was defined when all three of the following variables were below the cut-off point according to Centers for Disease Control and Prevention: ferritin $<15 \mu \mathrm{g} / \mathrm{l}$; $\mathrm{TS}<16 \%$; MCV $<85 \mathrm{fl}^{(32,33)}$. Maternal Fe deficiency anaemia (IDA) was defined in those cases with ID and $\mathrm{Hb}$ below $110 \mathrm{~g} / \mathrm{l}^{(32)}$. Reduced Fe stores in cord blood were defined when ferritin in cord blood was below $35 \mu \mathrm{g} / \mathrm{l}^{(34)}$. As presented previously $^{(29)}$, C-reactive protein (CRP) was only analysed in $<50 \%$ of the cases and we chose not adjust the Fe status measures for inflammation.

\section{Neurodevelopmental assessment}

Assessments of infant neurodevelopment at 18 months of age were conducted using the Bayley Scales of Infant Development, Third Edition (BSID-III). The BSID-III measures infant development across five domains: cognitive skills, receptive language, expressive language, fine motor development and gross motor development. The BSID-III was always performed in the research centre by the same trained psychologist (F. J. T.-E.). Mothers also filled in the BSID-III questionnaire assessing the infants' social-emotional behaviour.

\section{Statistics}

All statistical analyses were performed using the SPSS statistical software package for Windows (version 22.0; IBM SPSS Inc.). The subscales of Bayley scores were explored using a histogram and Q-Q plots and we assumed normal distribution as no significant skew was observed. Continuous variables were displayed as the mean values and standard deviations and explored using ANOVA, whereas categorical variables were compared using $\chi^{2}$ or Fisher's exact tests.

In the present analyses, we explored the association of Bayley scores at 18 months with the following risk factors: maternal Ow/Ob, maternal ID at 34 weeks and maternal ID at delivery. Maternal IDA at 34 weeks and at delivery as well as reduced $\mathrm{Fe}$ stores in cord blood were considered but not explored because of small sample sizes in the cases with registered Bayley scores. The three risk factors' univariate association with neurodevelopment was tested using $t$ test, comparing the mean value between those with $v$. those without risk (crude analyses). In a second step, the comparisons were adjusted for sex, gestational age at birth and maternal education level using ANCOVA as these correlated to at least one Bayley score or differed between the compared groups. Other possible confounders considered were maternal age and prevalence of breast-feeding. However, as they did not correlate to the Bayley results or differ significantly between the groups compared, they were not included in the adjusted models.

Third, maternal Ow/Ob and GD were introduced, one by one, in the ANCOVA models for maternal ID to explore independent associations. Similarly, presence of maternal ID at 34 weeks and at delivery were introduced in the ANCOVA models of maternal Ow/Ob.
The PREOBE project originally included four different groups, depending on maternal BMI and diabetic status (normal weight, Ow, Ob or GD). The pre-study power calculation aimed to detect differences in birth weight of $0.8 \%$ (assumed standard deviation $=1.5 \%$ ) between the different groups and the minimum group size for each group was set to thirty-seven motherchild pairs, assuming a drop-out rate of $25 \%$ until delivery ${ }^{(29)}$. For the present secondary analyses, no pre-study power calculation was performed.

\section{Results}

\section{Analysed subjects and their characteristics}

The study profile for the current study is presented in Fig. 1. We excluded seven cases from all analyses because of preterm birth ( $n$ 5) or congenital disorders diagnosed after birth $(n 2)$. The drop-out rate was low before delivery ( $n 21$ (6\%)) but following a large dropping out between birth and 18 months ( $n 109$ (33\%)), a sample of 194 children were assessed using the Bayley test at 18 months. The reasons for dropping out were mostly not reported by the mothers. Unfortunately, information about maternal Fe status at 34 weeks and at delivery were not available in all cases analysed for Bayley scores. The background and baseline characteristics and the prevalence of maternal ID and IDA of the analysed cases are presented in Table 1.

\section{Effects of iron status on neurodevelopment}

The results of the analyses of Bayley scores compared between ID and non-ID cases are presented in Tables 2 and 3. The offspring from mothers with ID at 34 weeks had significantly lower composite motor scores at 18 months of age. Maternal ID at delivery was negatively associated with lower receptive, expressive and composite language scores as well as with lower cognitive scores at 18 months. All associations remained significant after adjustment for background confounders and for GD. The correlation between maternal ID at delivery and the cognitive and language scores at 18 months all remained significant even when adjusting for maternal Ow/Ob.

\section{Effects of overweight/obesity}

We have previously presented data of Bayley scores using an alternative stratification and sample ${ }^{(30)}$. In that study, we found that offspring of obese, non-diabetic mothers showed a trend of lower scores in motor development and no differences in language and cognitive scores at 18 months. In the present analyses, we compared the offspring of mothers who were $\mathrm{Ow} / \mathrm{Ob}$ with those of normal-weight mothers and explored the effect, adjusted for maternal ID (Table 4). The results showed significantly lower gross motor scores in this group. The differences remained significant after adjusting for sex, gestational age at birth, for maternal education level and also in statistical models adjusting for ID at 34 weeks and ID at delivery, respectively. 
Fig. 1. Study profile. This observational study included 331 pregnant women, 166 who were overweight or obesity $\left(\mathrm{BMI} \geq 25 \mathrm{~kg} / \mathrm{m}^{2}\right)$ and seventy-nine with gestational diabetes. Iron status was analysed in 283 and 184 cases, respectively, at 34 weeks of gestation and at delivery. In total, twenty-one cases dropped out before delivery and another 109 did so before the follow-up study. ${ }^{*}$ Overall, seven cases were excluded because of congenital disorders diagnosed after birth ( $n$ 2) or because of preterm birth $(n 5)$.

\section{Discussion}

In the present study, we explored how maternal ID and maternal Ow/Ob associated with Bayley scores of the offspring at 18 months of age. We found that composite motor scores were negatively associated with maternal ID at 34 weeks of gestation, and cognitive and language scores were negatively associated with maternal ID at delivery. The associations with ID at delivery remained both after adjusting for confounders and when adjusting for maternal $\mathrm{Ow} / \mathrm{Ob}$ and GD. Presence of maternal $\mathrm{Ow} / \mathrm{Ob}$ correlated negatively to another subscale at 18 months, the gross motor scores. The fact that the associations between maternal characteristics and Bayley scores at 18 months, in most cases, remained significant after adjusting for each other suggests that both maternal ID and maternal Ow/Ob contribute to a risk for impaired neurodevelopment.

$\mathrm{Fe}$ is essential for optimal brain function, being involved in neurotransmitter synthesis, cellular metabolism and myelination of neurons ${ }^{(1,10)}$. There is convincing evidence from previous trials that ID in fetal life and early infancy are associated with poor neurodevelopment ${ }^{(5-7,35)}$. Nevertheless, the exact risk contribution of ID at different severity and doses to different stages of brain development is still unclear, particularly, in human research ${ }^{(1,6,24)}$.
Because of high Fe requirements in both the fetus and the mother, maternal ID is a common condition in pregnancy ${ }^{(36,37)}$. The rationale for identifying it as a risk factor in infant neurodevelopment is that the fetus receives reduced placental transfer of Fe, causing either reduced Fe stores for the important 1st month of postnatal brain development, when Fe requirements in the infant are largely met by the inborn stores ${ }^{(24,38)}$, or direct prenatal compromise during fetal brain development ${ }^{(6,9,39)}$. The magnitude of this risk has previously been considered low, because of the efficient Fe uptake mechanism in the placenta. However, this has been questioned over the last few years, as several studies have shown that fetal Fe stores can still be compromised when the mother is Fe deficient ${ }^{(12-16)}$. Further, Lozoff et $a l .{ }^{(17)}$ recently showed in a randomised controlled trial that Fe supplementation to a pregnant woman at risk for ID, improved the $\mathrm{Fe}$ stores in the offspring until 9 months of age.

Although limited by its observational design, our results support the hypothesis that maternal ID during the third trimester constitutes a possible risk for sub-optimal brain development in the offspring. Few studies explored the association between maternal ID and offspring neurodevelopment. A recent micronutrient supplementation trial in pregnant women in rural China found that infants born to mothers with prenatal IDA had a lower mental development index until 
Table 1. Background and baseline characteristics in 183 mother-child pairs analysed in the present study

(Mean values and standard deviations; numbers and percentages)

\begin{tabular}{|c|c|c|c|}
\hline & $n$ & Mean & SD \\
\hline \multicolumn{4}{|l|}{ Maternal characteristics } \\
\hline Maternal age at study entry (years) & 183 & 31.9 & $4 \cdot 3$ \\
\hline Education at university level & 183 & & \\
\hline $\begin{array}{l}n \\
\%\end{array}$ & & \multicolumn{2}{|c|}{$\begin{array}{c}85 \\
46 \cdot 4\end{array}$} \\
\hline Previous siblings & 182 & \\
\hline $\begin{array}{l}n \\
\%\end{array}$ & & \multicolumn{2}{|c|}{$\begin{array}{c}83 \\
45 \cdot 6\end{array}$} \\
\hline Maternal pre-gestational BMI $\left(\mathrm{kg} / \mathrm{m}^{2}\right)$ & 183 & $26 \cdot 0$ & $5 \cdot 1$ \\
\hline Maternal Ow/Ob & 183 & & \\
\hline$n$ & & \multicolumn{2}{|c|}{90} \\
\hline$\%$ & & \multicolumn{2}{|c|}{$49 \cdot 2$} \\
\hline Gestational diabetes & 183 & \\
\hline$n$ & & \multirow{2}{*}{\multicolumn{2}{|c|}{$\begin{array}{c}44 \\
24 \cdot 0\end{array}$}} \\
\hline$\%$ & & & \\
\hline Fe supplementation at entry & 163 & \multirow{2}{*}{\multicolumn{2}{|c|}{56}} \\
\hline$n$ & & & \\
\hline$\%$ & & \multicolumn{2}{|c|}{$34 \cdot 4$} \\
\hline \multicolumn{4}{|l|}{ Maternal Fe status } \\
\hline Ferritin at 34 weeks $(\mu \mathrm{g} / \mathrm{l})$ & 177 & $16 \cdot 7$ & 8.9 \\
\hline $\mathrm{Hb}$ at 34 weeks $(\mathrm{g} / \mathrm{l})$ & 179 & $116 \cdot 7$ & $12 \cdot 4$ \\
\hline MCV at 34 weeks (fl) & 179 & $86 \cdot 7$ & 4.9 \\
\hline Transferrin saturation at 34 weeks (\%) & 178 & $16 \cdot 8$ & $10 \cdot 8$ \\
\hline Ferritin below $15 \mu \mathrm{g} / \mathrm{l}$ at 34 weeks & 177 & \multirow{2}{*}{\multicolumn{2}{|c|}{82}} \\
\hline$n$ & & & \\
\hline$\%$ & & \multicolumn{2}{|c|}{$46 \cdot 3$} \\
\hline ID at 34 weeks & 175 & \multirow{2}{*}{\multicolumn{2}{|c|}{34}} \\
\hline$n$ & & & \\
\hline$\%$ & & \multicolumn{2}{|c|}{19.4} \\
\hline IDA at 34 weeks & 175 & \multirow{3}{*}{\multicolumn{2}{|c|}{$\begin{array}{c}18 \\
10 \cdot 3\end{array}$}} \\
\hline$n$ & & & \\
\hline$\%$ & & & \\
\hline Ferritin at delivery $(\mu \mathrm{g} / \mathrm{l})$ & 140 & 24.4 & $12 \cdot 2$ \\
\hline $\mathrm{Hb}$ at delivery $(\mathrm{g} / \mathrm{l})$ & 137 & $120 \cdot 3$ & 18.5 \\
\hline MCV at delivery (fl) & 137 & 85.9 & 5.4 \\
\hline Transferrin saturation at delivery (\%) & 140 & 17.4 & 11.9 \\
\hline Ferritin below $15 \mu \mathrm{g} / \mathrm{l}$ at delivery & 140 & \\
\hline$n$ & & & \\
\hline$\%$ & & \multicolumn{2}{|c|}{$\begin{array}{c}20 \\
16 \cdot 4\end{array}$} \\
\hline ID at delivery & 130 & & \\
\hline$n$ & & & \\
\hline$\%$ & & & \\
\hline IDA at delivery & 130 & & \\
\hline$n$ & & & \\
\hline$\%$ & & & \\
\hline Infant characteristics & & & \\
\hline Boys & 183 & & \\
\hline$n$ & & & \\
\hline$\%$ & & & \\
\hline Gestational age at birth (weeks) & 183 & 39.6 & $1 \cdot 1$ \\
\hline Birth length $(\mathrm{cm})$ & 169 & 50.5 & $2 \cdot 0$ \\
\hline Birth weight $(\mathrm{kg})$ & 183 & 3.33 & 0.43 \\
\hline Head circumference at birth $(\mathrm{cm})$ & 147 & 34.5 & 1.4 \\
\hline Apgar score at $5 \mathrm{~min}$ & 145 & 9.8 & 0.5 \\
\hline Exclusive breast-feeding at 3 months & 179 & & \\
\hline$n$ & & & \\
\hline$\%$ & & & \\
\hline Ferritin below $35 \mu \mathrm{g} / \mathrm{l}$ in cord blood & 79 & & \\
\hline$n$ & & & \\
\hline$\%$ & & & \\
\hline
\end{tabular}

Ow/Ob, overweight or obese $\left(\mathrm{BMI} \geq 25 \mathrm{~m} / \mathrm{kg}^{2}\right)$; MCV, mean cell volume; ID, Fe deficiency; IDA, Fe deficiency anaemia.

24 months of age, whereas there was no association with ID without anaemia ${ }^{(40)}$. In contrast to these results, we found a negative association between ID and neurodevelopment, even though the prevalence of IDA was relatively low among pregnant mothers. In fact, the prevalence of IDA even decreased between 34 weeks of gestation and delivery, most likely due to Fe supplementation, confirming that the explored cohort was at low risk for IDA.

A negative association between neurodevelopment and early stages of maternal ID is further supported by two recent animal models ${ }^{(41,42)}$ and from an observational study where the authors observed a significant association between maternal Fe intake and directional diffusion of water in the infant brain, measured using magnetic resonance ${ }^{(43)}$.

From a clinical perspective, the present study gives further support to the policy of general Fe supplementation programmes in pregnant women. Few randomised studies have assessed the effect of maternal Fe supplementation on longterm offspring neurodevelopment. One study, following the offspring of Australian mothers who were randomly assigned to Fe supplements or placebo during pregnancy, failed to show any positive effect on cognition at 4 years of age, or behaviour at $6-8$ years of age ${ }^{(44,45)}$. The mothers in the Australian trial were given general supplementation and the effects on neurodevelopment were not separately analysed for mothers with ID or IDA at baseline. Mainly on the basis of these Australian results, Cochrane, a consensus paper from the Committee on Nutrition of the European Society for Gastroenterology, Hepatology, and Nutrition (ESPGHAN) and a recent meta-analysis all concluded that there is no convincing evidence that Fe supplementation to a non-anaemic woman would improve neurodevelopment in the infant ${ }^{(18,19)}$. With regard to populations with a higher prevalence of ID and IDA, there are also few long-term follow-up studies available. The Chinese study above found no overall effect on Bayley scores following maternal supplementation ${ }^{(40)}$. We conclude, supported by our observation and the lack of previous data, that further long-term studies exploring the effect of Fe supplementation in different populations are warranted. In such studies, the possible adverse effects of Fe supplementation should be evaluated as well.

Our results with regard to the association between neurodevelopment and maternal $\mathrm{Ow}$ and $\mathrm{Ob}$ have been discussed in detail elsewhere ${ }^{(30)}$. In the present analyses we used an alternative approach of analysing the risk by adding all $\mathrm{Ow}$ and $\mathrm{Ob}$ mothers together, regardless of GD, and showed that this risk factor associated negatively with the gross motor score even after adjusting for gestational age, sex, maternal educational level and maternal ID.

This study was limited by its observational design, making possible causality difficult to explore. Confounding effects from non-monitored dietary, sociodemographic and genetic factors may be present. Further limitations were the lack of details regarding type, duration and dose of maternal Fe supplementations as well as a lack of inflammatory markers. There is a potential risk that a few cases of ID are incorrectly considered as non-ID because of concurrent inflammation. However, out of ninety-four CRP measures at 34 weeks of gestation, only one case had a CRP value above 5, suggesting a low risk for such a biased effect. Moreover, we did not adjust for the multiple testing, causing a possible risk for false positive findings, and there were a limited number of mothers participating in the 
Table 2. Bayley scores at 18 months of age compared between children born to mothers with and those without Fe deficiency (ID) at 34 weeks of gestation (Mean values and standard deviations)

\begin{tabular}{|c|c|c|c|c|c|c|c|c|}
\hline \multirow[b]{2}{*}{ Bayley scores at 18 months } & \multicolumn{2}{|c|}{ ID (34 weeks, $n$ 34) } & \multicolumn{2}{|c|}{ Non-ID (34 weeks, $n$ 141) } & \multirow[b]{2}{*}{$P_{1}^{*}$} & \multirow[b]{2}{*}{$P_{2} \dagger$} & \multirow[b]{2}{*}{$P_{3} \ddagger$} & \multirow[b]{2}{*}{$P_{4} \S$} \\
\hline & Mean & SD & Mean & SD & & & & \\
\hline Composite language & $106 \cdot 7$ & $8 \cdot 1$ & $106 \cdot 9$ & 8.5 & 0.898 & 0.780 & 0.939 & 0.747 \\
\hline Expressive language & $10 \cdot 6$ & 1.8 & $10 \cdot 6$ & 1.8 & 0.820 & 0.861 & 0.768 & 0.885 \\
\hline Receptive language & 11.6 & 1.4 & $11 \cdot 8$ & 1.5 & 0.442 & 0.326 & 0.376 & 0.296 \\
\hline Composite motor & $113 \cdot 3$ & 9.9 & $117 \cdot 1$ & $9 \cdot 2$ & $0.038 \|$ & $0.038 \|$ & 0.060 & $0.035 \|$ \\
\hline Fine motor & $12 \cdot 3$ & 1.8 & $13 \cdot 0$ & $2 \cdot 2$ & 0.084 & 0.055 & 0.055 & 0.058 \\
\hline Gross motor & $12 \cdot 0$ & 2.4 & $12 \cdot 6$ & $2 \cdot 0$ & 0.138 & 0.189 & 0.311 & 0.167 \\
\hline Composite cognitive & $118 \cdot 7$ & 10.9 & 121.6 & $11 \cdot 1$ & 0.165 & 0.170 & 0.147 & 0.169 \\
\hline Composite socio-emotional & $99 \cdot 6$ & $18 \cdot 1$ & 101.9 & $15 \cdot 5$ & 0.455 & 0.469 & 0.592 & 0.459 \\
\hline
\end{tabular}

${ }^{*} P_{1}$ value: for unadjusted difference $(t$ test $)$

$+P_{2}$ value: for differences adjusted for sex, maternal education and gestational age at birth (ANCOVA).

$\ddagger P_{3}$ value: for differences adjusted for sex, maternal education, gestational age at birth and maternal obesity/overweight (ANCOVA).

$\S P_{4}$ value: for differences adjusted for sex, maternal education, gestational age at birth and gestational diabetes (ANCOVA).

॥ Significant $P<0.05$.

Table 3. Bayley scores at 18 months of age compared between children born to mothers with and those without Fe deficiency (ID) at delivery (Mean values and standard deviations)

\begin{tabular}{|c|c|c|c|c|c|c|c|c|}
\hline \multirow[b]{2}{*}{ Bayley scores at 18 months } & \multicolumn{2}{|c|}{ ID delivery ( $n$ 10) } & \multicolumn{2}{|c|}{ Non-ID delivery $(n 120)$} & \multirow[b]{2}{*}{$P_{1}{ }^{*}$} & \multirow[b]{2}{*}{$P_{2} \dagger$} & \multirow[b]{2}{*}{$P_{3} \ddagger$} & \multirow[b]{2}{*}{$P_{4} \S$} \\
\hline & Mean & SD & Mean & SD & & & & \\
\hline Composite language & 99.5 & 8.6 & $107 \cdot 6$ & 8.3 & $0.004 \|$ & $0.004 \|$ & $0.008 \|$ & $0.004 \|$ \\
\hline Expressive lanquage & 9.0 & 1.8 & 10.6 & 1.9 & $0.008 \|$ & $0.013 \|$ & $0.022 \|$ & $0.011 \|$ \\
\hline Receptive language & $10 \cdot 8$ & 1.4 & 11.9 & 1.5 & $0.031 \|$ & $0.026 \|$ & $0.036 \|$ & $0.025 \|$ \\
\hline Composite motor & $115 \cdot 8$ & $12 \cdot 8$ & $116 \cdot 7$ & 9.5 & 0.789 & 0.780 & 0.996 & 0.779 \\
\hline Fine motor & $12 \cdot 2$ & $2 \cdot 0$ & $12 \cdot 8$ & $2 \cdot 2$ & 0.375 & 0.311 & 0.322 & 0.344 \\
\hline Gross motor & $13 \cdot 0$ & 2.5 & $12 \cdot 6$ & 2.0 & 0.584 & 0.544 & 0.303 & 0.591 \\
\hline Composite cognitive & $114 \cdot 0$ & 9.7 & 121.5 & $10 \cdot 9$ & $0.038 \|$ & $0.039 \|$ & $0.037 \|$ & $0.036 \|$ \\
\hline Composite socio-emotional & 96.0 & $12 \cdot 2$ & $102 \cdot 4$ & $16 \cdot 7$ & 0.242 & 0.256 & 0.393 & 0.259 \\
\hline
\end{tabular}

${ }^{*} P_{1}$ value: for unadjusted difference $(t$ test)

$+P_{2}$ value: for differences adjusted for sex, maternal education and gestational age at birth (ANCOVA).

$\ddagger P_{3}$ value: for differences adjusted for sex, maternal education, gestational age at birth and maternal obesity/overweight (ANCOVA).

$\S P_{4}$ value: for differences adjusted for sex, maternal education, gestational age at birth and gestational diabetes (ANCOVA).

II Significant $P<0.05$

Table 4. Bayley scores at 18 months of age compared between children born to mothers with and those without overweight or obesity $(\mathrm{Ow} / \mathrm{Ob})\left(\mathrm{BMl}>25 \mathrm{~kg} / \mathrm{m}^{2}\right)$ (Mean values and standard deviations)

\begin{tabular}{|c|c|c|c|c|c|c|c|c|}
\hline \multirow[b]{2}{*}{ Bayley scores at 18 months } & \multicolumn{2}{|c|}{ Ow/Ob $(n 58)$} & \multicolumn{2}{|c|}{ Non-Ow/Ob ( $n$ 67) } & \multirow[b]{2}{*}{$P_{1}^{*}$} & \multirow[b]{2}{*}{$P_{2} \dagger$} & \multirow[b]{2}{*}{$P_{3} \ddagger$} & \multirow[b]{2}{*}{$P_{4} \S$} \\
\hline & Mean & SD & Mean & SD & & & & \\
\hline Composite language & $106 \cdot 2$ & $7 \cdot 6$ & $108 \cdot 0$ & $9 \cdot 2$ & 0.370 & 0.160 & 0.178 & 0.388 \\
\hline Expressive language & 10.5 & 1.9 & $10 \cdot 7$ & 1.9 & 0.577 & 0.272 & 0.261 & 0.533 \\
\hline Receptive language & 11.7 & $1 \cdot 2$ & $12 \cdot 0$ & 1.7 & 0.390 & 0.288 & 0.354 & 0.535 \\
\hline Composite motor & $115 \cdot 3$ & $9 \cdot 3$ & 117.8 & $10 \cdot 4$ & 0.148 & 0.108 & 0.184 & 0.114 \\
\hline Fine motor & $12 \cdot 7$ & $2 \cdot 1$ & $12 \cdot 9$ & 2.4 & 0.724 & 0.672 & 0.984 & 0.807 \\
\hline Gross motor & $12 \cdot 3$ & $2 \cdot 0$ & $13 \cdot 0$ & $2 \cdot 1$ & $0.047 \|$ & $0.037 \|$ & $0.042 \|$ & $0.025 \|$ \\
\hline Composite cognitive & $120 \cdot 7$ & 11.9 & $121 \cdot 2$ & $10 \cdot 2$ & 0.799 & 0.670 & 0.897 & 0.971 \\
\hline Composite socio-emotional & 99.4 & 17.4 & 103.9 & $15 \cdot 9$ & 0.134 & 0.108 & 0.160 & 0.156 \\
\hline
\end{tabular}

ID, Fe deficiency.

* $P_{1}$ value: for unadjusted difference $(t$ test)

$\dagger P_{2}$ value: for differences adjusted for sex, maternal education and gestational age at birth (ANCOVA)

$\ddagger P_{3}$ value: for differences adjusted for sex, maternal education, gestational age at birth and maternal ID at 34 weeks of gestation (ANCOVA).

$\S P_{4}$ value: for differences adjusted for sex, maternal education, gestational age at birth and maternal ID at delivery (ANCOVA).

II Significant $P<0.05$.

follow-up investigation at delivery, making the study population different when comparing the risk contribution at different stages of gestation. Finally, cord blood ferritin was only available in less than half of the cases of maternal ID and we could not statistically evaluate if the negative associations between
Bayley scores and maternal ID was linked to infant ferritin levels. Nevertheless, the sample size in this prospective study was relatively high, there are good data from animal models for mechanistic explanations and the present analyses were adjusted for the most relevant confounders. Further, we have 
performed novel analyses including both maternal Fe status and metabolic risk factors. To our knowledge, this mother-child cohort is one of the largest to include both maternal and infant data collection. As discussed, our observations are in line with previously discussed hypotheses and contribute with important data for further large-scale intervention studies.

\section{Conclusions}

In this study, we found that ID in late pregnancy is associated with impaired motor, cognitive and language development in the offspring at 18 months of life. The associations with regard to language and cognitive scores were independent of background factors, maternal $\mathrm{Ow} / \mathrm{Ob}$ and GD, and possibly represented a causal correlation. Further, we found that maternal ID in late pregnancy did not explain the negative effect of $\mathrm{Ow} / \mathrm{Ob}$ observed on gross motor development. The results suggest that both maternal $\mathrm{Ow} / \mathrm{Ob}$ and maternal ID are negatively associated with child neurodevelopment but at different subscales and that they should be considered independently of each other. Consequently, the children of pregnant women with simultaneous $\mathrm{Ow} / \mathrm{Ob}$ and ID are particularly at a risk for poorer neurodevelopment because of a double burden of risk. Future research to verify and clarify the mechanisms underlying the observed associations are required, preferably including longterm neurodevelopmental follow-up studies. We believe that these results should be taken into account when considering whether to provide Fe supplementation for pregnant women in general and obese pregnant women in particular.

\section{Acknowledgements}

The authors thank the women and children who participated in the study and also the obstetricians, paediatricians and technicians of the EURISTIKOS team at the Department of Pediatrics at the University of Granada, Spain.

This study received grants from Spanish Ministry of Innovation and Science, Junta de Andalucía: Excellence Projects, ref: P06-CTS-02341; Spanish Ministry of Economy and Competitiveness, ref: BFU2012-40254-C03-01; and MyNewGut FP7 EU Project (grant agreement no. 613979). Further support was received from Abbott Nutrition, Granada, Spain. The first author received Postdoc scholarships from Henning and Johan Throne-Holst's foundation, The Swedish Society of Medicine and the Swedish Research Council for Health, Working life and Welfare (FORTE, ref. 2014-2648). The funders had no role in the design, analysis or writing of this article.

S. K. B. performed the statistical analyses and wrote the first draft. F. J. T.-E. was responsible for the neurodevelopmental data collection and performed all Bayley tests. F. J. T.-E., L. G.-V., M. T. S. and C. M.-Z. collaborated in the follow-up study of the children and in data collection and interpretations. C. P., R. R., M. P. G. and H. J. M. participated in the study design and interpretations of data. C. C. was the principal investigator, responsible for the design and coordination of the research. She had the primary responsibility for the final content. All authors endorsed the data and conclusions and approved the final paper.

The authors declare that there are no conflicts of interest.

\section{References}

1. Georgieff MK (2007) Nutrition and the developing brain: nutrient priorities and measurement. Am J Clin Nutr 85, 614S-620S.

2. Anjos T, Altmae S, Emmett P, et al. (2013) Nutrition and neurodevelopment in children: focus on NUTRIMENTHE project. Eur J Nutr 52, 1825-1842.

3. Tomalski P \& Johnson MH (2010) The effects of early adversity on the adult and developing brain. Curr Opin Psychiatry 23, 233-238.

4. Cusick SE \& Georgieff MK (2012) Nutrient supplementation and neurodevelopment: timing is the key. Arch Pediatr Adolesc Med 166, 481-482.

5. Beard JL (2008) Why iron deficiency is important in infant development. J Nutr 138, 2534-2536.

6. Georgieff MK (2008) The role of iron in neurodevelopment: fetal iron deficiency and the developing hippocampus. Biochem Soc Trans 36, 1267-1271.

7. Lozoff B, Beard J, Connor J, et al. (2006) Long-lasting neural and behavioral effects of iron deficiency in infancy. Nutr Rev 64, S34-S43; discussion S72-S91.

8. Berglund SK, Westrup B, Hagglof B, et al. (2013) Effects of iron supplementation of LBW infants on cognition and behavior at 3 years. Pediatrics 131, 47-55.

9. Georgieff MK, Landon MB, Mills MM, et al. (1990) Abnormal iron distribution in infants of diabetic mothers: spectrum and maternal antecedents. J Pediatr 117, 455-461.

10. Radlowski EC \& Johnson RW (2013) Perinatal iron deficiency and neurocognitive development. Front Hum Neurosci 7, 585 .

11. Berglund S \& Domellof M (2014) Meeting iron needs for infants and children. Curr Opin Clin Nutr Metab Care 17, 267-272.

12. Lubach GR \& Coe CL (2006) Preconception maternal iron status is a risk factor for iron deficiency in infant rhesus monkeys (Macaca mulatta). J Nutr 136, 2345-2349.

13. De Pee S, Bloem MW, Sari M, et al. (2002) The high prevalence of low hemoglobin concentration among Indonesian infants aged 3-5 months is related to maternal anemia. J Nutr 132, 2215-2221.

14. Jaime-Perez JC, Herrera-Garza JL \& Gomez-Almaguer D (2005) Sub-optimal fetal iron acquisition under a maternal environment. Arch Med Res 36, 598-602.

15. Colomer J, Colomer C, Gutierrez D, et al. (1990) Anaemia during pregnancy as a risk factor for infant iron deficiency: report from the Valencia Infant Anaemia Cohort (VIAC) study. Paediatr Perinat Epidemiol 4, 196-204.

16. Allen LH (2000) Anemia and iron deficiency: effects on pregnancy outcome. Am J Clin Nutr 71, 1280s-1284s.

17. Lozoff B, Jiang Y, Li X, et al. (2016) Low-dose iron supplementation in infancy modestly increases infant iron status at 9 mo without decreasing growth or increasing illness in a randomized clinical trial in rural China. J Nutr 146, 612-621.

18. Domellof M, Braegger C, Campoy C, et al. (2014) Iron requirements of infants and toddlers. J Pediatr Gastroenterol Nutr 58, 119-129.

19. Peña-Rosas JP, De-Regil LM, Garcia-Casal MN, et al. (2015) Daily oral iron supplementation during pregnancy. Cochrane Database Syst Rev, issue 7, CD004736.

20. Neggers YH, Goldenberg RL, Ramey SL, et al. (2003) Maternal prepregnancy body mass index and psychomotor development in children. Acta Obstet Gynecol Scand 82, 235-240.

21. Brion MJ, Zeegers M, Jaddoe $\mathrm{V}$, et al. (2011) Intrauterine effects of maternal prepregnancy overweight on child cognition and behavior in 2 cohorts. Pediatrics 127, e202-e211. 
22. Van Lieshout RJ (2013) Role of maternal adiposity prior to and during pregnancy in cognitive and psychiatric problems in offspring. Nutr Rev 71, Suppl. 1, S95-S101.

23. Van Lieshout RJ, Taylor VH \& Boyle MH (2011) Pre-pregnancy and pregnancy obesity and neurodevelopmental outcomes in offspring: a systematic review. Obes Rev 12, e548-e559.

24. Cao C \& O'Brien KO (2013) Pregnancy and iron homeostasis: an update. Nutr Rev 71, 35-51.

25. Aigner E, Feldman A \& Datz C (2014) Obesity as an emerging risk factor for iron deficiency. Nutrients $\mathbf{6}, 3587-3600$.

26. Burke RM, Leon JS \& Suchdev PS (2014) Identification, prevention and treatment of iron deficiency during the first 1000 days. Nutrients 6, 4093-4114.

27. Nikonorov AA, Skalnaya MG, Tinkov AA, et al. (2015) Mutual interaction between iron homeostasis and obesity pathogenesis. J Trace Elem Med Biol 30, 207-214.

28. Phillips AK, Roy SC, Lundberg R, et al. (2014) Neonatal iron status is impaired by maternal obesity and excessive weight gain during pregnancy. J Perinatol 34, 513-518.

29. Berglund SK, Garcia-Valdes L, Torres-Espinola FJ, et al. (2016) Maternal, fetal and perinatal alterations associated with obesity, overweight and gestational diabetes: an observational cohort study (PREOBE). BMC Public Health 16, 207.

30. Torres-Espinola FJ, Berglund SK, Garcia-Valdes LM, et al. (2015) Maternal obesity, overweight and gestational diabetes affect the offspring neurodevelopment at 6 and 18 months of age a follow up from the PREOBE cohort. PLOS ONE 10, e0133010.

31. Acosta D, Balsells M, Ballesteros M, et al. (2015) Care of pregnancies complicated by diabetes. Clinical practice guidelines: 2014 update [Spanish]. Avances en Diabetología 31, 45-59.

32. Centers for Disease Control and Prevention (1998) Recommendations to prevent and control iron deficiency in the United States. MMWR Recomm Rep 47, 1-29.

33. Milman N, Bergholt T, Byg KE, et al. (2007) Reference intervals for haematological variables during normal pregnancy and postpartum in 434 healthy Danish women. Eur J Haematol 79, 39-46.

34. Siddappa AM, Rao R, Long JD, et al. (2007) The assessment of newborn iron stores at birth: a review of the literature and standards for ferritin concentrations. Neonatology 92, 73-82.
35. Tamura T, Goldenberg RL, Hou J, et al. (2002) Cord serum ferritin concentrations and mental and psychomotor development of children at five years of age. $J$ Pediatr 140, $165-170$.

36. McLean E, Cogswell M, Egli I, et al. (2009) Worldwide prevalence of anaemia, WHO Vitamin and Mineral Nutrition Information System, 1993-2005. Public Health Nutr 12, 444-454.

37. Cantor AG, Bougatsos C, Dana T, et al. (2015) Routine iron supplementation and screening for iron deficiency anemia in pregnancy: a systematic review for the U.S. Preventive services task force. Ann Intern Med 162, 566-576.

38. Oski FA (1993) Iron deficiency in infancy and childhood. $N$ Engl J Med 329, 190-193.

39. Golub MS \& Hogrefe CE (2015) Fetal iron deficiency and genotype influence emotionality in infant rhesus monkeys. J Nutr 145, 647-653.

40. Chang S, Zeng L, Brouwer ID, et al. (2013) Effect of iron deficiency anemia in pregnancy on child mental development in rural China. Pediatrics 131, e755-e763.

41. Geng F, Mai X, Zhan J, et al. (2015) Impact of fetal-neonatal iron deficiency on recognition memory at 2 months of age. J Pediatr 167, 1226-1232.

42. Tran PV, Dakoji S, Reise KH, et al. (2013) Fetal iron deficiency alters the proteome of adult rat hippocampal synaptosomes. Am J Physiol Regul Integr Comp Physiol 305, R1297-R1306.

43. Monk C, Georgieff MK, Xu D, et al. (2016) Maternal prenatal iron status and tissue organization in the neonatal brain. Pediatr Res 79, 482-488.

44. Zhou SJ, Gibson RA, Crowther CA, et al. (2006) Effect of iron supplementation during pregnancy on the intelligence quotient and behavior of children at $4 \mathrm{y}$ of age: long-term follow-up of a randomized controlled trial. Am J Clin Nutr $\mathbf{8 3}$, 1112-1117.

45. Parsons AG, Zhou SJ, Spurrier NJ, et al. (2008) Effect of iron supplementation during pregnancy on the behaviour of children at early school age: long-term follow-up of a randomised controlled trial. Br J Nutr 99, 1133-1139. 